\title{
Characteristic of a Proactive Assembly System
}

\author{
Kerstin Dencker ${ }^{1,2}$, Johan Stahre ${ }^{3}$, Åsa Fasth ${ }^{3}$, Peter Gröndahl ${ }^{1}$, Lena Mårtensson ${ }^{4}$ and Thomas Lundholm ${ }^{5}$ \\ ${ }^{1}$ Royal Institute of Technology, Department of Production engineering \\ ${ }^{2}$ Swerea IVF, Industrial Research \& Development Corp. \\ ${ }^{3}$ Chalmers University of Technology, Division of Production Systems \\ ${ }^{4}$ Royal Institute of Technology, Department of Industrial economics and management \\ ${ }^{5}$ Royal Institute of Technology, Centre for Design and Management of Machining Systems
}

\begin{abstract}
Competitive assembly systems must cope with frequent demand changes, requiring drastically shortened resetting and ramp-up times. Characteristics of assembly systems capable of rapid change are e.g. Flexibility; Robustness, Agility, and ability to handle frequent changes and disturbances. This paper proposes proactivity as a vital factor of semi-automated assembly systems to increase speed of change. Proactive systems utilize the full potential of human operators and technical systems. Such systems have ability to dynamically change system automation levels, resulting in decrease of time consumed for assembly tasks. Proactivity criteria for assembly systems are reviewed based on theory and industrial case studies
\end{abstract}

\section{Keywords:}

Assembly System, Proactive, Automation

\section{INTRODUCTION}

Present practices for development, design and use of assembly systems may not be adapted to the needs and future challenges of industry. Competitive systems for manufacturing, specifically assembly systems, will have to cope with increasingly frequent changes of product variants as well as increased variation in production volumes. At the same time, cost efficiency and "leanness" require appropriate quality, lower cost, and strong focus on value-adding activities. Preferably, product customization should be made as late as possible in the value-adding chain, i.e. mass customization [1]. Such demands require assembly systems, which is reliable, have high availability and have ability to produce the right product correctly. This means a combination of short resetting time and robustness of the system as whole and its resources. A major challenge is to reduce and minimize the lead-time that direct has influence on order-todelivery time, while maintaining flexibility and robustness to absorb late requirement market changes. This includes throughput time and cycle times for individual assembly processes. Also, there is a need to minimize resetting time between batches, time to repair, time for disturbance handling, and time to prepare for new variants or products in the assembly system. How can a radical reduction in the time needed to fulfil an assembly sequence be achieved?

The objective of the ProAct project is to identify proactive solutions for time minimization at operational shop floor level in assembly systems. The approach is based on the concept of proactivity; taking action by causing change towards a state and not only reacting to change when it happens [2]. ProAct is a collaborative effort involving six Swedish industries and three Swedish technical universities.

\section{BACKGROUND}

To minimize the time factors suggested, while remaining competitive, assembly system behaviour has to be proactive and that requires highly transformable system resources.

Dencker et al. [3] suggest that a proactive assembly system has the ability to prepare for:

- changes and disturbances during operation

- planned and long-term changes, and sustainable evolution of an assembly system

Main features required to prepare the assembly system are:

- flexibility

- robustnessspeed of change

- $\quad$ ability to handle frequent changes

- evolvability

In terms of system resources, we suggest that the proactive assembly system constitutes an integrated combination of the competence of "knowledge workers" [4] information, and automation. The proactive assembly concept was presented by Dencker et al. [5], along with arguments for focus on levels of automation, information, and competence as main drivers for the degree of proactivity.

A Proactive Systems should be able to respond quickly to a dynamically changing environment in real-time contexts. Such a system should also have the ability to act proactively and preventive before a situation emerges to a confrontation or crisis.

Proactivity should be employed in two different time perspectives; to be able to act proactively during operation and to maintain the proactive ability over time. The operation perspective involves manual, semi-automated, and automated operations and tasks to be handled proactively. The second perspective focuses on continuous changes to 
the system, in order to absorb disturbances and changing requirements.

\section{THE PROACTIVE ASSEMBLY SYSTEM STRUCTURE}

\subsection{System model}

According to Chapanis [6], a system is an interacting combination at any level of complexity, of people, material, tools, machines, software, facilities, and procedures designed to work together for some common purpose.

\section{System features Available resources System behaviou}

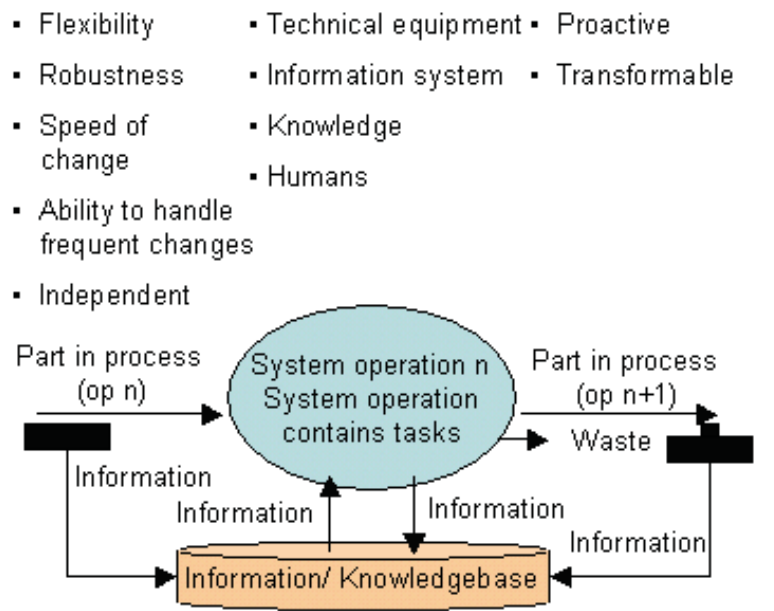

Figure 1 Theoretic model of an assembly system operation

Tharumarajah et al [7] describes that Self-organised systems can be developed through a Bionic Manufacturing System (BMS) protomodel [8]. Self-regularized and dependent systems can be used by applying Holonic Manufacturing System concepts [9]. The difference between evolvability in an operationally and managerially dependent and independent (BMS) system is that a dependent system is able to evolve in one general direction with internal congruence. For the independent system, each subsystem possess has that ability, making the whole system far more agile and responsive to internal and environmental changes. According to [10, 11], an Evolvable assembly system is a system which is "being based on many simple, reconfigurable, task-specific elements (system modules), this allows for a continuous evolution of the assembly system. "The proactive assembly system idea is to add skills from a human "agent" as a core assembly system resource, this will make the system more independent and it will receive a larger range by independence.

Development of semi-automated assembly system structures is generally framed by technical, ergonomic, and human work requirements. These contribute with constraints for the system structure. De Toni et al. [12] describes system process flexibility as 'the ability to produce a given set of part types', see figure 1. There are two distinct ways of being flexible for a given dimension of change and time period, i.e. range flexibility and mobility flexibility according to Upton [13]. Range number is a strict numerical count of the number of possible options that a system or resource can achieve. Mobility, or transformability, is defined as the ease or effort with which the system moves from one state to another. For each change of state within a specific range, transformability is assessed via transition penalties.

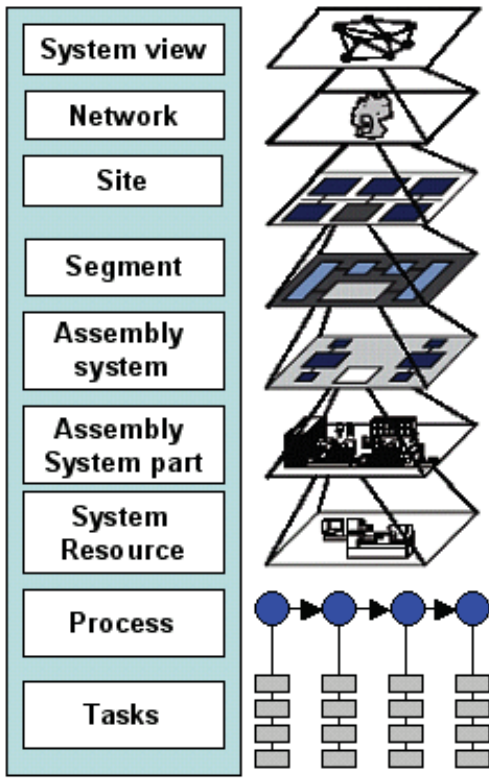

Figure 2 System process definition

The definition of assembly system flexibility focuses on the range flexibility and transformability that is described as the set of options or alternative process performance in a predefined dimension and aims for a more operational and managerial independence, see definition below. The transformability is the ability to move between optional states or system configurations. State-changes should be performed with a minimum of transition penalties, e.g. setup time for reconfiguration or re-routing.

Bjelkemyr [14] defines operational and managerial dependence (a) and independence (b) that can be seen in figure $3 \mathrm{In}$ the left view, the tinted sectors signify that each subsystem is unable to function satisfactory on its own, i.e. it is operationally dependent on the other sectors to create a whole. The striped circle in the middle signifies that they all have one joint management looking after the whole system.
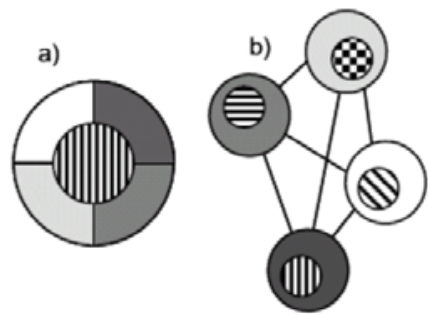

Figure 3 operational and managerial dependence (a) and independence (b)

Independent and constituent systems are useful in their own right and generally operate independent of other systems.

The independent system has a larger range but the transformability must be agile if the system should be considered as efficient. The Reconfigurable Manufacturing Systems paradigm [15] is an example of predefined dependent- range flexibility and fast transformability. Robustness is the ability to handle predictable and 
unpredictable variations with minimal loss of functionality causing penalty time. It determines the range of magnitudes of changes within which the feasible respond occur. Low values of transition penalties imply agile transformability in the system and transition penalties are dependent on time parameters.

\subsection{System resources}

Resources in an assembly system are:

\section{Technical equipment}

Automation that is flexible and quickly adjustable to different levels of automation in the assembly system. This applies to mechanical/physical as well as information/cognitive levels of automation. [16-18]

Information system

Efficient and dynamic flow of predictable as well as unpredictable information between assembly, product development, production planning preparation, suppliers, marketing etc. [19-20]

\section{Knowledge}

The system knowledge, facts and methods, as the knowledgebase carried by humans or computer.

\section{Human operators}

Operator team that have the knowledge of system tasks and competence to perform demanded knowledge based task. Quick, precise, and efficient competence development for assembly operators

The ISO definition SS 624070 [21] defines competence as Ability and willingness to carry out a task by applying knowledge and skills. When defining competence the following implications have been to the words used: Ability experience, comprehension and judgment to use knowledge and skills in practice, where willingness is the attitude, commitment, courage and responsibility; knowledge means facts and methods -to know and skills is to carry out in practice - to do.

Resources as they are defined in figure 3, include human resources considered as specific means with a given capability and a given capacity. Those means are considered as being able to be involved in the manufacturing process through assigned tasks. That does not include any modelling of an individual or common behaviour of human resource excepted in their capability to perform a given task in the manufacturing process (e.g.: transformation of raw material or component, provision of logistic services). That means that human resources are only considered, as the other, from the point of view of their functions, their capabilities and their status (e.g. idle, busy). That excludes any modelling or representation of any aspect of individual or common «social» behaviour. By adding the concept of ability to vary level of automation [22] the range of possible system solutions becomes higher but puts new requirements on the competence of the operator team and the information. This Level of Automation concept was defined in Frohm [23]. To realize the productivity potential of automated equipment human involvement is needed [5] to support technical equipment. As stated, the combination of human and machine skills and the possibility to allocate tasks is widening the range of possibilities. Relevant human involvement will be different depending on the situation and the assembly system design. The information system must provide assembly systems with correct and essential information, contributing to the operators' decision support.

\subsection{System transformation and System tasks}

Ability to vary levels of information, levels automation and competence has earlier been identified as three important factors in a proactive assembly system [3]. The system transformation process defines the tasks that should be performed during set up and the task allocation for desired processes. Such an assembly system needs improved information exchange and this requires an adequate information and decision support system. [19]. Further, the system needs competent operators with ability and mandate to make decisions concerning short term planning, recourse allocation and actions in disturbance handling. Proactive assembly system require a team of operators that are able to exercise their own judgment, "think by themselves", proceed on their own initiatives, and anticipate future problems, in order to avoid exceptional production-, or work situations. The tasks performed by the proactive assembly system are e.g. performing assembly tasks, short-term planning, real-time recourse allocation and actions in disturbance handling. The assembly system task are structured by using Stahre, [24] planning, programming, monitoring, performing, intervening, and learning. For the proactive assembly system to achieve and maintain an acceptable level of performance considering time parameters defined on section 1 , the interaction between human and machine demands that the collaboration of systems must correspond to a set of tasks that the operators and/or machine are able to perform at any time. These include the demand to perform the operations included in the process, and to response within the constraints provided by time and system operating requirements. To achieve this correspondence, the design must be based on a thorough analysis of the tasks included in the operations, meaning the tasks that are supposed to be carried out in the process. This includes how the tasks will be carried out in practice and what resources the system has like information, e.g. task description, and competence.

The system transformation process should be as short as possible over a running time during a continuous product variation flow. According to Rasmussen's [25] task definition the skill and expertise of the operator affect the human behaviour, which can be categorized in Rasmussen's three levels of performance: skill, rule-, and knowledge-based (SRK) behaviour [25].

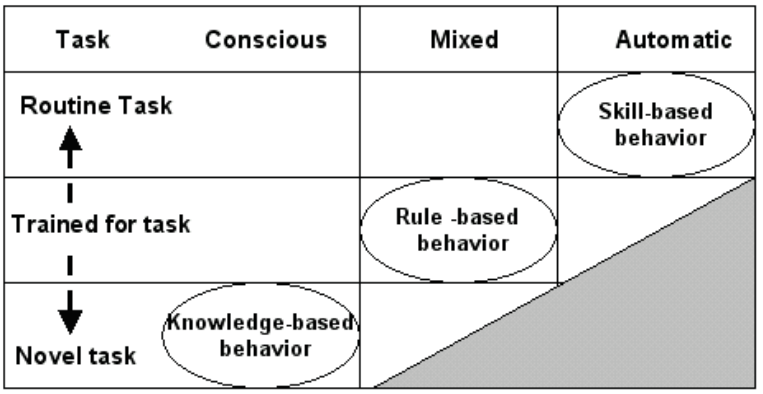

Figure 4 Rasmussen SRK form a task perspective

Each level of the SRK taxonomy defines different ways of representing the constraints in the environment and thus different level of cognitive control of the operator, see figure 
4. The skill-based behaviour takes place without conscious attention or control and consists of smooth, automated, and highly integrated patterns. Rule-based behaviour is characterized by pattern matching with stored rules derived from previous successful experiences. At the rule-based level people are aware of their cognitive activities, and hence, can verbalize the used rules. The knowledge-based behaviour is required in unfamiliar situations and demands a conscious, focal attention of the operator. In these situations previous experience is no longer valid and a solution must be improvised by functional reasoning. The task analyze

\section{TASK ALLOCATION}

The allocation of tasks has traditionally been based on rather simple principles, which tend to consider the system in terms of its parts rather than as a whole. The left-over principle means that tasks that have not been automated, due to either technical or economical reasons, are assigned to the humans in the system. The compensatory principle, also referred to as Fitts' list, uses a list or table of the strong and weak features of humans and machines as a basis for assigning functions and responsibilities to the various system components [26]. More recently, a complimentarily principle for the allocation of functions has been advocated. Instead of focusing on the capabilities and limitations of the components in the system, the focus is on how the humans and machines can complement and support each other. Human and machine functions are not seen as being in competition or as being replaceable, but as being mutually dependent and necessary to achieve the overall purpose-Joint System [27]. The allocation of tasks should thus serve to maintain control of the situation and support the retaining of human skills according to Grote [28]

The possibilities to dynamically allocate tasks between human and technical resources are required. What do we expect from the system and what duties, tasks and actions will the system be responsible for e.g. information handling, communication and decision making in normal operations and occurrences (predicted and unpredicted)? Instead of having the view that the operator is a user of the system the humans and the machines are resources within the system, figure 3. A structured analysis of the skills of a human "agent" as a core assembly system resource should be an integrated part of system development. To support the idea of resource allocation the system tasks has been defined from a SRK view but only by its character not assuming that the task is allocated to the human.

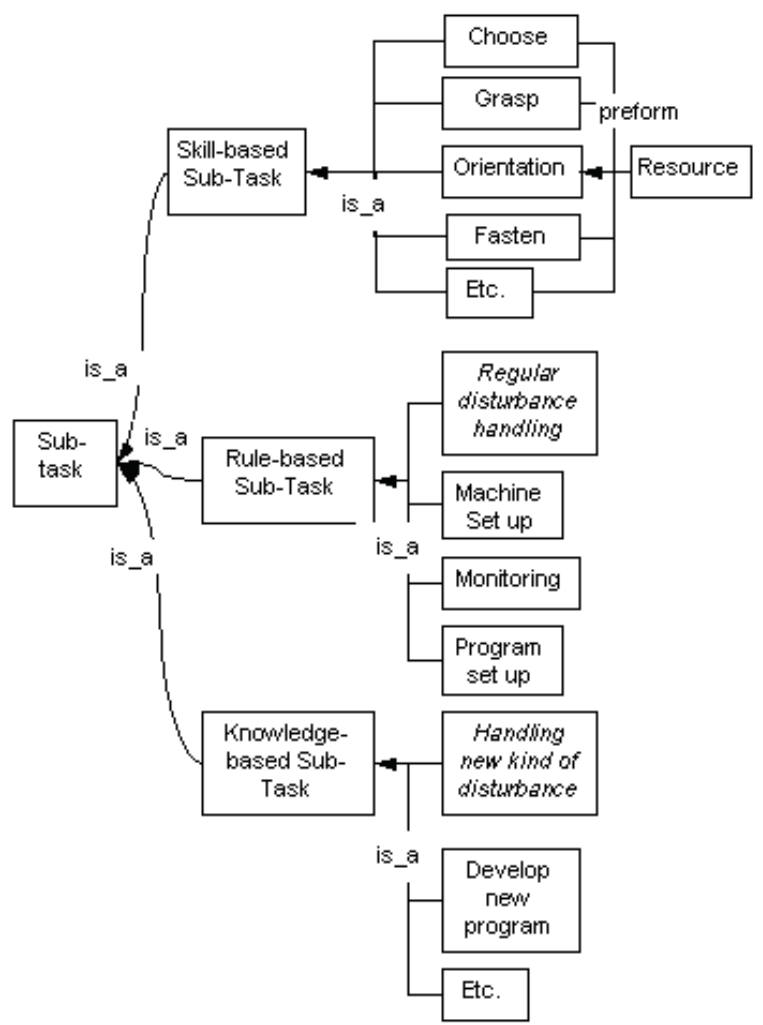

Figure 5 Categorization of assembly tasks according to Rasmussen SRK

\section{INDUSTRIAL CASE STUDIES}

As previous suggested, To enhance of understanding the relation of LOA and proactivity four case studies was performed during autumn 2007 at four industrial companies with final assembly in Sweden. The case studies focused on the easies of the defined tasks in an assembly system, perform assembly task. The measurement of LoA was conducted with a methodology for level of automation assessment called DYNAMO [22]. In the case studies evaluated the time parameters according to in relation of LoA. The purpose was to detect time difference of performing the task assembly according to different competence. For a proactive assembly system a task like perform assembly defined as a skill-based task but the surveys defined that the individual operator performance time was dependant on experience according to the contribution to lead time. Though the competence for the assembly skill-based tasks goes from low to high in a short time if the instructions of the tasks support novel operators.

Given a fixed competence in the operator group and a fixed Lol an increase of LoA at first decreases the time to perform the task, investigated system are represented by varying LoA solutions illustrated in fig 6. Frohm [22] argues however, that when the system reaches an increased LoA the effects of automation deviates negative from the expected because of e.g. breakdowns. According to Harlin et al. [28] is the condition of realizing the potential of automation to decrease the negative effects caused by system complexity originate from high LoA. 
Solutions of varying LoA outside recourse property boundary
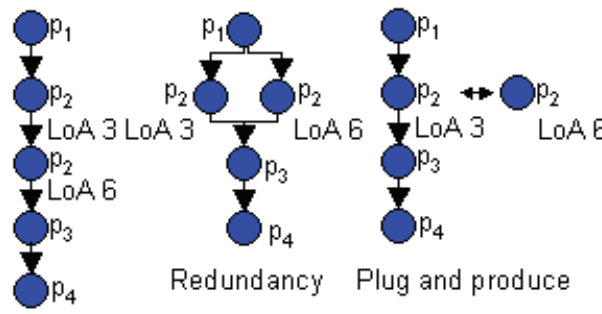

Redundancy Plug and produce

In line replacement

Figure 6 Examples of investigated LoA solutions.

According to the survey the situation with fixed competence in the operator group and fixed LoA but the possibility to vary the amount of information gave reduction in the setup time as well as the task time. The decreased setup time is a result of when the assembly system knew ahead what products that in the queue their made a last minute optimization of the future setups within predefined frames. They where able to change the order of the next ten orders and the sequence was delimited of product similarities. A product that had to many similar but not same components, was not allowed to be assembled after each other because of the high mistake rate. The possibility to rearrange between the orders gave possibilities to make the setup times shorter and more setup was performed as external setup. The higher amount of information to the operator team gave the effect that if a machine did not fulfil the requirement the group allocated the desired task to an operator until the machine got repaired.

\section{DISCUSSION}

Proactive system behaviour may not be described in terms of classic hierarchical manufacturing system models. A modern assembly system must be independent operational and managerial to support an agile transformation of the resources. Given this independency, tasks can be reallocated proactively. However our studies show that tasks cycle time are affected by Lol, LoA, LoC and their interrelations. The results shows that there is a connection between the parameters LoA, Lol and LoC. Naturally; the lead-time is directly related to the operator's experience to perform the assembly task. This also influences potential proactivity. Further development of tools for defining LoC and Lol will give the possibility to measure the variation of all three parameters in real time. By enabling task reallocation in real time, decreased operational times like setup and task time will be possible. The LoA concept gives the system a wider range of possible solutions but the range can be delimited by the level of competence of the operator group when it comes to difficult tasks like disturbance handling and reconfiguration.

\section{CONCLUSION}

The proposed proactive approach for structuring future, semiautomated assembly systems is a strategic solution for integrating human and technical resources in an efficient system. It is not the person, nor the machine that is capable of proactivity, but rather the fully integrated system of human and technical resources. Thus, proactive systems will decrease the lead time in assembly. By reducing time parameters in the chosen areas; LoA, LoC and Lol. It is our belief that interrelated adjustment and transformability received by ability to vary the level of automation, the level of information, and the level of competence will result in a radical decrease of time consumed for assembly tasks.

\section{ACKNOWLEDGMENTS}

The research is financially supported by the Swedish Agency for Innovation Systems (VINNOVA) as it is a part of the ProAct project (http://proact.iip.kth.se/). The authors would like to thank the companies participating in the ProAct project.

\section{REFERENCES}

[1] Jackson, M., An analysis of flexible and reconfigurable production systems: an approach to a holistic method for the development of flexibility and reconfigurability. Linköping, Univ., 2000, Lindköping, Sweden ISBN 917219-797-8.

[2] Cambridge Advanced Learner's Dictionary, 2008.

[3] Dencker, K., Stahre, J., Gröndahl, P., Mårtensson, L. Lundholm, T., and Johansson C., 2007, An approach to proactive assembly systems - towards competitive assembly systems, IEEE international symposium on assembly and manufacturing (ISAM), University of Michigan, Ann Arbor, US.

[4] Drucker, P. F,1999, Management Challenges for the $21^{\text {st }}$ Century. Harper Business, USA.

[5] Dencker, K., Stahre, J., Gröndahl, P., Mårtensson, L., Lundholm, T., Bruch, J. and Johansson C., 2007, Proactive assembly systems - realizing the potential of human collaboration with automation, In proceedings of the IFAC-CEA, cost effective automation in networked product development and manufacturing, Monterrey, Mexico.

[6] Chapanis, A., 1996, Human factors in systems engineering, New York: John Wiley \& Sons, Inc.

[7] Tharumarajah, A., Welles, A.J., Nemes, L., 1996, Comparison of the bionic, fractal and holonic manufacturing system concepts, International Journal Computer Integrated Manufacturing, 9 (3) pp. 217-226.

[8] Okino, N., 1980, Bionic Manufacturing Systems (BMS) Bio-Modelon based systems design. CAM-I, Annual International Conference, New Orleans (US).

[9] Bell, R., Rahimifard, S. and Toh, K.T.K., 1998. Holonic systems, in: Handbook of Life Cycle Engineering:Concepts, Models and Technologies, Molina, A., Sanchez, J.M., Kusiak, A., (Eds), 115-150, Ed. Kluwer Academic Publishers, London, UK.

[10] Lindberg, B., Onori, M., Semere, D., 2007, Evolvable Production System a position paper, In Swedish Production Symposium, Gothenburg, Sweden.

[11] Semere, D., Barata, J. and Onori, M., 2007. Evolvable Production Systems, Developements and Advances, tbp. IEEE Int. Symposium on Assembly and Manufacturing. Ann-Arbor, US.

[12] De Toni, A., Tonchia, S., 2005, Definitions and linkages between operational and strategic flexibilities, Omega, 33:525-540.

[13] Upton, D.M., 1996, What Really Makes Factories Flexible? In: Hayes, R.H., Pisano, G.P., Upton, D.M. (eds), Competing Through Capabilities, The Free Press, New York, US. 
[14] Bjelkemyr, M., Semere, D., Lindberg, B., 2007, An Engineering system perspective of System of Systems Methodology, 1st Annual IEEE Systems Conference, Honolulu, HI, US.

[15] Mehrabi, M.G, Ulsoy, A.G, Koren, Y., 2000, Reconfigurable Manufacturing Systems: Key to Future Manufacturing, Journal of Intelligent Manufacturing, Vol. 11, No. 4, pp 403-419.

[16] Fasth, A., Stahre, J. and Dencker, K., to be submitted 2008, Analysing changeability and time parameters due to levels of Automation in an assembly system, In FAIM, Skövde, Sweden.

[17] Fasth, Å., Frohm, J. and Stahre, J., 2007, Relations between Performers/parameters and level of automation, In IFAC workshop on manufacturing modelling, management and control Budapest, Hungary.

[18] Fasth, A.., Stahre, J. and. Dencker, K., to be submitted 2008, Measuring and analysing Levels of Automation in an assembly system, In The 41st CIRP conference on manufactoring systems, Tokyo, Japan.

[19] Bruch, J. Karltun, J., Johansson, C., and Dencker, K., A proactive assembly work setting - Information requirements, to be submitted 2008. In The 41st CIRP Conference on Manufacturing Systems Tokyo, Japan.

[20] Bruch, J., Johansson, C., Karltun, J. and Winroth, M., 2007, Considering design demands of a proactive assembly system - a position paper, In Swedish Production Symposium, Sweden.

[21] SIS/SS/624070, 2003, Competence management systems - requirement. ICS 03.120.10.
[22] Frohm, J., 2007, Levels of Automation in Production Systems, Department of Production Development, Production System, Chalmers University of Technology, Gothenburg, Sweden.

[23] Frohm, J., Lindström and Bellgran, M., 2005, A model for parallell levels of automation within manufacturing, In proceedings of the 18th International Conference on Production Research, Italy.

[24] Stahre, J., 1995, Towards Human Supervisory Control in Advanced Manufacturing Systems, Doctoral Thesis, Chalmers University of Technology Sweden.

[25] Rasmussen, J., 1983, Skills, Rules, Knowledge, Signals, Signs and Symbols and other Distinctions Human Performance Models, IEEE Transactions on Man, Systems and Cybernetics, SMC-13/3:pp. 257266.

[26] Fitts, P. M., Ed., 1951. Human Engineering for an Effective Air Navigation and Traffic-Control System, Columbus, Ohio State University Research Foundation, US.

[27] Hollnagel, E., Woods, D., Levenson, N., et al., 2006, Resilience Engineering. Ashgate, Hamp-shire, England.

[28] Grote, G. 2004, Uncertainty management at the core of system design, Annual Reviews in Control 28, pp. $267-$ 274.?

[29] Harlin, U., Frohm, J., Berglund, M. and Stahre J., 2006, Towards efficient automation implementation. In: Mayer, F. And stahre, J. (Eds) Proceedings of the 9th symposium IFAC on Automated System based on Human Skills and Knowledge, Nancy, France. 


\title{
Virtual Manufacturing Work Systems
}

\author{
Peter Butala ${ }^{1}$, Ivan Vengust ${ }^{1,2}$, Rok Vrabič ${ }^{1}$, Lovro Kuščer ${ }^{1}$ \\ ${ }^{1}$ Department of Control and Manufacturing Systems, University of Ljubljana, Ljubljana, Slovenia \\ ${ }^{2}$ PS d.o.o., Logatec, Slovenia
}

\begin{abstract}
Contemporary computer and information technologies enable digitalization and virtualization of real systems and their exploration in a virtual space. In the paper, a virtual CNC machine tool is presented. It is build up to support development of CNC controllers and control algorithms on the basis of the Hardware-in-the-Loop principle, where the object of control is substituted with a virtual one. The developed digital model of a machine tool may serve also as a model of a digital factory.

In the case study the virtual model of a desktop CNC engraving machine LAKOS 150 is described. The model is integrated in the control loop with a real CNC controller via the CNC2VML interface, which converts the controller's signal into digital information and vice versa. This enables communications between the CNC controller and the virtual machine tool model in real-time. The model is visualized in a 3D graphical environment. The applied programming techniques are generic and based on the open architecture principle and standard graphic library.
\end{abstract}

Keywords:

Digital model, CNC machine tool, Mechatronic system design, Hardware-in-the-loop, Virtualization

\section{BACKGROUND AND MOTIVATION}

Digitalization and virtualization of work systems open new perspectives for development and operations of complex manufacturing work systems. A virtual work system model is an effective tool for demonstration of complex work structures and their control and operational principles. The virtual model embeds explicit knowledge, which can be explored and reused. For example, users can investigate and make experiments with the virtual model by themselves and thus can gain better understanding and can learn much more naturally and effectively.

The contribution reveals development of a virtual CNC machine tool. The objective of the research is to develop a digital model (1) as a building block of a digital factory for simulation of machine operations and (2) for visualization of machine tool behavior for remote control, educational purposes and, last but not least (3) for development of a real CNC controller based on the hardware-in-the-loop principle. Several other applications of a virtual model of a machine tool are possible [1, 2].

The virtual model of a desktop CNC engraving machine LAKOS 150 is described as an example.

\subsection{Digital factory}

Manufacturing nowadays relies on computer controlled work systems and computer aided technologies, which are more or less integrated in a complex cybernetic structure - a factory. Development and operations of such structures open challenging engineering and managerial issues, which have to be addressed with adequate methods based on knowledge.

The digital factory represents an approach to explicit formulation of manufacturing knowledge and it's coding into software. The objective here is to efficiently support design, development and operations of a real factory. The digital factory is a set of digital models and computer aided tools for design of new manufacturing systems and planning of production for new products [3]. In the future, production of any new product will be examined through simulations before its realization in a real environment. This will significantly contribute to better decisions in the development process, increased quality of solutions, accelerated development and decreased development costs. In order to build up an integrated digital model of a real factory, digital models of individual work systems are needed.

\subsection{Hardware-in-the-loop principle}

A typical manufacturing work system is a mechatronic system, which is composed of a complex electro-mechanical structure, i.e. an object of control, and a controller. The controller is composed of control hardware elements and highly specialized control software.

In design and development of a new work system, the object of control as well as the controller hardware and software have to be developed concurrently and then integrated in a prototype. In early development phases, neither the object of control nor the controller elements are available. The problem that arises from this fact is that the control software has to be developed on the basis of a conceptual solution and its specification, which is as a rule incomplete and a matter of change. The software also cannot be tested on the target hardware during the development. Hence, inadequate solutions and bugs in the software are common and have to be resolved in the integration phase.

The Hardware-in-the-Loop (HiL) principle enables much more effective concurrent development of a mechatronic system. The principle is based on substitution of a real object of control in a control loop with its software model [4].

Figure 1 shows a typical HiL system structure. The object of control is substituted by its digital object. If one introduces prototype hardware, the control loop can be closed already at the very beginning of its development. 